УДК 004.62

\title{
СЕРВИС ОNLINE ПОИСКА ПОСТАВЩИКОВ КАК СРЕДСТВО ОПТИМИЗАЦИИ БИЗНЕС-ПРОЦЕССОВ МАЛЫХ И СРЕДНИХ ПРЕДПРИЯТИЙ
}

\author{
Новикова Александра Владимировна \\ канд. экон. наук, доцент, \\ доцент кафедры «Отраслевая экономика» \\ ФГБОУ ВО «Брянский государственный \\ технический университет»
}

\begin{abstract}
Аннотация. Основными результатами исследования являются анализ рынка решений по поиску поставщиков, разработка концепции сайта для поиска поставщиков, адаптированного под потребности малого и микробизнеса, а также обоснование экономической эффективности проекта.

Ключевые слова: поставщики, покупатели, оптимизация, малый и средний бизнес, платформа.
\end{abstract}

\author{
ONLINE SUPPLIER SEARCH SERVICE \\ AS A MEANS OF OPTIMIZING BUSINESS PROCESSES \\ SMALL AND MEDIUM-SIZED ENTERPRISES
}

Novikova Alexandra Vladimirovna Candidate of Economic Sciences, Associate Professor

\begin{abstract}
The main results of the research are the analysis of the market of solutions for the search for suppliers, the development of a site concept for the
\end{abstract}


search for suppliers, adapted to the needs of small and micro-businesses, as well as the justification of the economic efficiency of the project.

Key words: suppliers, buyers, optimization, small and medium business, platform.

Объектом исследования выступают технологии поиска поставщиков онлайн, а предметом исследования - разработка платформы по быстрому и удобному поиску поставщиков товаров и услуг для малого и среднего бизнеса через интернет.

Цель исследования - проанализировать опыт эксплуатации уже имеющихся на рынке продуктов по поиску поставщиков, их особенности, достоинства и недостатки и предложить свое решение, учитывающее специфику работы с малым (в том числе микро-) и средним бизнесом.

К методам проведенного исследования можно отнести: сравнительный анализ и синтез, сбор и обработку статистических данных, моделирование, прогнозирование

Бизнес - это сложная система, и успех того или иного предприятия зависит от синергии множества факторов [1]. Одним из ключевых факторов является выбор подходящих поставщиков. Каким бы ни был бизнес: магазин, салон красоты, автосервис или даже небольшое производство, он рано или поздно сталкивается с проблемой поиска поставщиков материалов, услуг, оборудования.

В наше время эта проблема становится всё более актуальной. Согласно динамике социально-экономического развития Брянской области, количество малых и средних предприятий в 2018 году составило 13200 единиц, а их суммарный оборот оценивается в 298,83 млрд рублей, что на 9,2\% больше, чем в 2017 году. В 2017 году количество малых и средних предприятий составило 13035 единиц, а оборот по всем видам 
деятельности - 273,56 млрд. рублей, что на 20,2\% больше, чем в 2016 году [2].

Для того чтобы найти статистику за 2019 год, обратимся к данным Брянскстата. Так, численность микропредприятий (юридические лица) составила 10245, а их оборот оценивается в 124,5 млрд рублей (+7\% к уровню 2018 года). Численность малых предприятий составила 1370 единиц, а их суммарный оборот - 138,2 млрд рублей $(+0,5 \%$ к уровню 2018 года). Таким образом, наблюдается устойчивый рост.

Однако в масштабах России ситуация с малым и средним бизнесом складывается не лучшим образом. Количество предприятий сокращается, а вклад в экономику падает. Согласно данным бизнес-портала РБК, главными проблемами предприниматели называют высокие издержки при работе с поставщиками товаров и услуг, а также высокие налоги и социальные отчисления.

К сожалению, не каждому предпринимателю хватает знаний и опыта для того, чтобы найти нужного поставщика, и при этом не разориться и не нарваться на мошенников [3].

На помощь приходят современные информационные технологии [4]. Предлагается создать онлайн платформу, которая поможет потребителям найти оптимального, то есть максимально подходящего по всем критериям поставщика, а поставщикам найти покупателей на свои товары и услуги.

Для достижения этой цели планируется решить ряд задач:

1) проанализировать уже имеющиеся на рынке продукты;

2) определить достоинства и недостатки этих продуктов, а также особенности работы с ними;

3) разработать концепцию собственного решения, основываясь на особенностях работы с малыми и средними предприятиями, учитывая опыт уже имеющихся систем; 
4) обосновать экономическую эффективность проекта и спрогнозировать период окупаемости.

Научная новизна проекта заключается в комплексном исследовании рынка сервисов для поиска поставщиков и покупателей online, a также разработке принципиально нового решения, ориентированного на малый и средний бизнес. Реализация проекта позволит уменьшить издержки предпринимателей на поиск поставщиков для своего бизнеса, тем самым способствуя увеличению деловой активности сначала в Брянской области, которую предлагается рассматривать в качестве пилотного региона, а затем и по всей России.

\section{1. Анализ конкурентной среды}

По данным портала «supple.biz» в России насчитывается 1,3 млн поставщиков, работающих с юридическими лицами. Это очень много. При таком большом предложении бизнесу достаточно трудно найти подходящий вариант.

Сейчас процесс поиска поставщика необходимого товара или услуги у большинства малых и средних компаний происходит следующим образом: директор или специалист по закупкам через поисковую систему находит интересующие его предложения и обзванивает потенциальных поставщиков.

Есть и альтернативные варианты. Можно искать поставщика на различных выставках и форумах, но постоянно посещать такие мероприятия может себе позволить далеко не каждая компания в силу дороговизны. Можно искать поставщика в печатных изданиях (газетах, журналах, каталогах). Однако поиск всеми указанными способами - это достаточно долгий и трудоёмкий процесс, который к тому же не всегда приводит к желаемым результатам.

Большинство поставщиков на рынке - это посредники или дистрибьюторы. То есть организации, занимающиеся покупкой и 46 
перепродажей товаров. Иногда товар проходит несколько итераций куплипродажи, чтобы в итоге попасть к конечному потребителю, поэтому одной из главных проблем является величина наценки на товар от многочисленных посредников [5].

Конечно, искать поставщика лучше напрямую, поскольку это более надежно и выгодно. Отечественный производитель представлен практически во всех секторах. Это может быть как малый и средний, так и крупный бизнес. Не секрет, что непосредственные производители предлагают более долгосрочное сотрудничество на более выгодных условиях, чем дистрибьюторы [6]. Однако, как правило, непосредственные производители напрямую с малым и средним бизнесом не работают, так как это экономически нецелесообразно. Крохотные заказы малого бизнеса незначительны в рамках промышленных объемов. Поэтому и возникает проблема поиска. В то же время выбор подходящего поставщика является одним из ключевых факторов эффективного функционирования предприятия [7].

В связи с этим особую важность для малых и средних предприятий приобретает проблема максимально удобного и быстрого поиска надёжных поставщиков напрямую без дистрибьюторов [8].

На данный момент в интернете существует несколько отечественных платформ, предоставляющих услуги по поиску поставщика online. Это «Dropo», «Поставщики.ру», «ОптЛист». Рассмотрим каждую из них подробнее.

Dropo - платформа поиска поставщиков для интернет-магазинов. Dropo самостоятельно ищет поставщиков и отбирает по своим критериям «интересные» товары. Предпочтение отдается производителям и оптовым поставщикам. 
Dropo самостоятельно заключает договоры с поставщиками, то есть является классическим дистрибьютором. Клиент в виде владельца небольшого интернет-магазина размещает у себя на сайте каталог товаров с сайта Dropo. Бесплатный пакет включает в себя каталог на 1000 товаров и выгрузку на сайт не более 10 товаров. Далее интернет-магазину остается только принять оплату у покупателя и передать еe Dropo. Всю дальнейшую работа с клиентом (доставка, обслуживание, возврат товара) ведет уже Dropo.

Однако есть нюансы. Интернет-магазин может работать только с Dropo, то есть параллельно работать ещё с несколькими платформами или поставщиками нельзя. К тому же появляется существенная наценка, иногда превышающая цену у оптового поставщика в 2 раза.

Проанализировав отзывы в интернете, выяснилось, что предприниматели часто жалуются на проблемы с доставкой товаров. Доставка или переносится, или сервис заявляет, что больше не работает с этим поставщиком (причем выясняется это уже после перевода платежа Dropo). В результате покупатели отказываются от заказа, а интернет-магазин несет как финансовые, так и репутационные издержки.

«Поставщики.ру» представляет собой платформу, позволяющую поставщикам и покупателям находить друг друга. Поставщики размещают на сайте свои товары, а покупатели - свои заказы. Платформа является посредником между поставщиком и покупателем и не несёт никаких обязательств.

Рассмотрим сайт со стороны покупателя. Товары отсортированы очень грубо, то есть присутствует общая категория, например «Мототовары», в которой представлено все, что теоретически относится к товарам для мотоциклов. Более точной сортировки, даже по региону, к сожалению, нет, что создает существенные неудобства. Поиск также не помогает, так как в 
нем вообще отсутствует какая-либо сортировка. Платформа позволяет без регистрации узнать контактный номер поставщика, его сайт и электронный адрес, что является неоспоримым преимуществом. Сайт позволяет оставлять отзывы о товарах, но этим никто не пользуется. К тому же ни у продавцов, ни у товаров нет хотя бы простейшей рейтинговой системы [9].

Для того, чтобы разместить свой товар, необходимо пройти бесплатную регистрацию организации, после чего можно размещать неограниченное количество товаров в рамках выбранных категорий.

«ОптЛист» позволяет поставщикам размещать товары и услуги, а покупателям свои заказы. Платформа также является посредником.

Сначала рассмотрим сайт со стороны покупателя. Платформа нацелена на поиск непосредственно поставщиков, а не на поиск товаров, поэтому на сайте нет разделения товаров по категориям и какого-либо поиска. То есть если клиенту нужны компьютерные колонки, то он ищет не сами колонки, а поставщика периферийного оборудования. Связаться с поставщиком можно только через сайт. Однако многие поставщики на сайт не заходили очень давно, то есть выяснить актуальную цену и как-то связаться с ними не представляется возможным. По многим поставщикам в интернете нет никакой информации, кроме данных о государственной регистрации, поэтому отсутствие на сайте рейтинговой системы - это большой недостаток.

Зарегистрироваться на сайте как поставщик можно бесплатно, однако позиция в поиске будет самой низкой, что делает регистрацию по сути бессмысленной. Для того чтобы подняться в рейтинге, нужно покупать подписку. Чем дороже подписка, тем выше место в рейтинге.

Таким образом, были изучены три наиболее популярных в Рунете сервиса по поиску поставщиков. При изучении подобных платформ был обнаружен ряд общих недостатков: 
1) отсутствие качественного поиска по товарам: на всех сайтах товары очень грубо распределены по базовым категориям, а какая-либо сортировка внутри этих категорий отсутствует;

2) невозможность отсортировать поставщиков по региону, например, если клиент хочет найти поставщика в своем регионе и не платить за доставку;

3) отсутствие понятной рейтинговой системы и системы отзывов;

4) нет возможности сортировать товары (по цене, рейтингу);

5) нацеленность на крупные оптовые продажи [10].

Ко всему прочему, количество поставщиков на этих сайтах оставляет желать лучшего, и нацелены они в основном на крупный бизнес (минимальное количество заказываемого товара в среднем составляет от 1000 до 10000 ед.). Таким образом, можно сделать вывод о том, что вышеуказанные платформы не в полной мере подходят малому и среднему бизнесу.

В связи с этим предлагается создать всероссийский реестр поставщиков товаров и услуг в виде веб-сайта и мобильного приложения, в котором были бы устранены вышеуказанные недостатки. Это позволит малому и среднему бизнесу быстро находить наиболее выгодные для себя варианты всего за пару кликов.

\section{2. Характеристика проекта}

Для начала четко определим целевую аудиторию проекта. Это преимущественно микро- и малый бизнес, но сервис будет полезен и среднему бизнесу. Сфера деятельности предприятий не так важна. Платформа будет ориентирована на мелкие оптовые заказы.

Поставщик должен будет зарегистрировать свою компанию в системе. Перед тем, как разрешить поставщику публиковать свои предложения, будет проводиться его проверка по базам данных ЕГРЮЛ/ЕГРИП, ФНС, POССТАТ, ПФР, ФСС (информацию предоставит сервис «ЗАЧЕСТНЫЙБИЗНЕС»). 
У каждого поставщика будет своя страница. На этой странице будет размещаться краткая информация о компании, сведения о государственной регистрации, краткая статистическая информация (такую информацию предоставляет целый ряд сервисов, например «СБИС»), история работы с сервисом (дата регистрации, число выполненных заказов, средняя оценка от заказчиков). На этой же странице будет располагаться каталог товаров.

Для того, чтобы начать пользоваться сервисом, покупатель также должен будет зарегистрироваться в системе и пройти проверку. После этого появится возможность просматривать полную информацию о поставщике и вести с ним переписку. Если клиент захочет узнать полную информацию о поставщике, он должен будет приобрести подписку.

В предыдущем разделе к основным недостаткам уже существующих платформ было отнесено отсутствие отзывов и рейтинговой системы у товаров. Это планируется исправить. Для успешной работы рейтинговой системы каждый заказ должен быть зарегистрирован. Для этого после завершения сделки заказчик должен будет отметить факт совершения сделки на сайте. В целях соблюдения коммерческой тайны в системе необходимо будет оформить только сам факт совершения сделки по конкретному товару, без каких-либо подробностей. Поставщик должен подтвердить факт сделки. После этого заказчику будет предложено оценить по пятибалльной шкале качество товара и уровень сервиса поставщика. Оценка будет проводиться по нескольким критериям:

1) качество товара, соответствие заявленных характеристик реальным;

2) качество работы с поставщиком: честность и открытость поставщика, соблюдение условий договора, достоверность запрашиваемой информации и др.;

3) дополнительные услуги, например собственная служба доставки (если их нет, то этот пункт можно пропустить) или рассрочка; 
4) соблюдение сроков поставки товара или услуги (соблюдение сроков передачи товара курьерской службе и т.д.).

Поставщику также будет предложено оценить своего покупателя. Оценка будет проводиться по нескольким критериям:

1) качество работы с клиентом: честность и открытость клиента, соблюдение условий договора, достоверность запрашиваемой информации и др.;

2) соблюдение сроков оплаты: своевременность проведения платежа, отсутствие просрочки;

3) дополнительные преимущества работы с клиентом, например, оплата в день совершения сделки и т.д.

Рейтинговая система является основой платформы, выгодно отличающей её от конкурентов, поэтому требование оставлять отзыв для каждого заказа будет обязательным условием работы с платформой. У каждого товара, поставщика и покупателя будет свой рейтинг, что позволит существенно сократить время и затраты на поиск надёжного поставщика и качественного товара или услуги, а продавцам существенно сэкономить время и нервы и сразу отказываться от сотрудничества с «нерадивым» клиентом.

Важным недостатком уже существующих платформ является отсутствие качественного поиска по товарам, то есть внутри основных категорий (например «Товары для сада и огорода») нет дополнительных подкатегорий (как в любом интернет-магазине). То есть в разделе «Товары для сада и огорода» будет представлено все: и лопаты, и триммеры, и качели, и семена. Это очень неудобно и непрактично. Поиск не исправляет ситуацию. Например, клиенту нужны доски длиной в 1 метр. Поиск по запросу «доска 1 метр» скорее всего не даст результата, а на некоторых платформах (например «ОптЛист») в принципе отсутствует поиск по товарам. В связи с чем 
планируется создать полноценный каталог товаров, как в крупных интернетмагазинах (Ebay, Ozon). Плюс к этому добавить возможность сортировки товаров (по способу оплаты, региону, цене и т.д.).

При организации поиска предпочтение будет отдаваться региональным поставщикам. То есть первыми будут выводиться предложения именно от местных производителей и поставщиков.

К главным особенностям предлагаемой платформы можно отнести, во-первых, ориентацию на мелкий опт, а во-вторых, ориентацию на непосредственных производителей и дилеров, которые либо являются эксклюзивными представителями производителя в регионе, либо занимаются прямыми поставками из-за границы. Ориентация платформы на мелкий опт означает то, что в результатах поиска платформа будет отдавать предпочтение поставщикам, работающих с небольшими партиями товара (10-100 ед.).

Таким образом, предлагаемая методика организации поиска поможет существенно «продвинуть» небольших региональных производителей, а рейтинговая система позволит в значительной мере упростить процесс взаимодействия между поставщиком и заказчиком, что, несомненно, положительно скажется на динамике развития бизнеса, особенно в условиях всеобщей цифровизации и перевода бизнес-процессов в сеть.

Бизнес-модель проекта предлагается организовать следующим образом. Доступ к базе данных поставщиков для клиентов будет предоставляться по подписке. Для новых пользователей предусмотрен пробный период в 30 дней. Планируется возможность приобрести подписку на месяц, полгода и год. Для поставщиков регистрация и работа на сайте будет бесплатной в определённых границах. Плата будет взиматься за добавление новых сфер деятельности, сверх тех, что были указаны при 
регистрации и при превышении количества выставленных товаров (больше $50)$.

\section{3. Расчет экономической эффективности}

Для реализации проекта планируется привлечение собственных средств и средств фонда Президентских грантов.

Реализация проекта требует значительных инвестиций. Примерная структура затрат по категориям представлена в табл. 1-3.

Таблица 1

\section{Смета разработки и создания проекта в виде сайта} (единовременные затраты)

\begin{tabular}{|c|c|}
\hline Наименование статьи расходов & Цена, тыс. руб. \\
\hline Дизайн сайта (индивидуальный, 1 вариант) & 15 \\
\hline Верстка сайта (адаптивная) & 5 \\
\hline Система управления & 11,5 \\
\hline Создание внутренней структуры сайта \\
(более 100 страниц) \\
\hline Регистрация пользователей (расширенная)
\end{tabular}

Продолжение таблицы 1 


\begin{tabular}{|c|c|}
\hline Мультивалютность (3 валюты) & 4,5 \\
\hline $\begin{array}{c}\text { Пересчет цен по курсу } \\
\text { (синхронизация с ЦБ) }\end{array}$ & 4 \\
\hline Импорт/экспорт контента & 6 \\
\hline Поиск по каталогу (текстовый) & 3 \\
\hline Модуль «рекомендованные товары» & 6 \\
\hline Поиск по сайту (с подсказками) & 2 \\
\hline Комментарии к публикациям & 3,5 \\
\hline на основе плагинов ВК и Ғасеьоо) & 6,5 \\
\hline Рейтинг публикаций & 4 \\
\hline Отзывы & 3 \\
\hline Онлайн-консультант & 150 \\
\hline Рекламная система & 284 \\
\hline Покупка сервера & \\
\hline Итого: & \\
\hline
\end{tabular}

Таблица 2

\section{Постоянные затраты}

\begin{tabular}{|l|c|}
\hline \multicolumn{1}{|c|}{ Наименование статьи расходов } & Цена, тыс. руб. \\
\hline ЗП системного администратора & 35 \\
\hline Юридические услуги на аутсорсинге & 40 \\
\hline Бухгалтерские услуги на аутсорсинге & 30 \\
\hline
\end{tabular}

Продолжение таблицы 2 


\begin{tabular}{|l|c|}
\hline \hline Домен & 2 \\
\hline 3/п штатного программиста & 50 \\
\hline $\begin{array}{l}\text { Страховые взносы } \\
\text { Амортизация сервера } \\
\text { (планируется замена раз в год) }\end{array}$ \\
\hline Итого: & 12,567 \\
\hline
\end{tabular}

Таблица 3

Переменные затраты

\begin{tabular}{|l|c|}
\hline \multicolumn{1}{|c|}{ Наименование статьи расходов } & Цена, тыс. руб. \\
\hline Реклама & 500 \\
\hline Канцелярия & 2 \\
\hline Маркетинг & 200 \\
\hline Итого & 702 \\
\hline
\end{tabular}

Стоимость размещения продукции компании на сайте - 2,5 тыс. руб.

Планируемое количество размещений в месяц - 300 .

Стоимость доступа по подписке - 500 руб./месяц.

Количество подписчиков в месяц - 400.

Планируемая выручка в месяц: 925 тыс. руб.

Далее рассчитаем срок окупаемости проекта.

Ежемесячная чистая прибыль: (925 $000-172067-702000) * 0,8=40$ 746,4 руб.

Чистая прибыль в год: $40746,4 * 12=488$ 956,8 руб.

Если инвестиции в проект составляют 284 тыс. руб., срок окупаемости составит 284 000/488 956,8=7,2 месяца. 
Итак, каждый предприниматель сталкивается с проблемой поиска надежных поставщиков. Особенно остро эта проблема ощущается в текущей крайне нестабильной экономической обстановке. Поэтому важно оптимизировать все бизнес-процессы и сделать каждый аспект работы бизнеса максимально эффективным. К одному из ключевых направлений, остро нуждающихся в оптимизации, можно отнести построение процесса взаимоотношений с поставщиками.

В результате работы над проектом была предложена концепция сайта, где представители микро-, малого и среднего бизнеса могли бы найти подходящего под их критерии поставщика товаров и услуг. Кроме того, сайт позволит самим поставщикам находить клиентов с минимальными рисками.

Проведенный анализ показал, что имеющиеся на текущий момент платформы не в полной мере удовлетворяют требованиям микро- и малого бизнеса. К основным недостаткам этих платформ можно отнести: отсутствие качественного поиска по товарам, невозможность отсортировать поставщиков по региону, отсутствие понятной рейтинговой системы и системы отзывов, невозможность сортировки товаров, и самое главное нацеленность на крупные оптовые продажи.

Концепция предлагаемого проекта предполагает решение вышеуказанных проблем. Качественная организация поиска, нацеленность на работу с региональными поставщиками и мелкооптовыми партиями товаров, а также разработка рейтинговой системы должны привлечь внимание широкого круга предпринимателей из разных отраслей экономики.

В связи с тем, что проект направлен на оптимизацию одного из самых сложных и трудозатратных аспектов работы бизнеса, его внедрение позволит бизнесу существенно сократить риски, и тем самым подстегнет к развитию малый и средний бизнес не только в Брянской области, которая рассматривается в качестве пилотного региона, но и во всей России. 


\section{Список литературы}

1. Исайченкова, В. В. Укрепление стратегической позиции предприятия с помощью механизма оптимизации бизнес-процессов / В. В. Исайченкова // European Social Science Journal. - 2014. - № 10-2 (49). C. 59-66.

2. Прогноз социально-экономического развития Брянской области на 2019 год и на плановый период 2020 и 2021 годов. [Электронный ресурс]. Режим доступа: http://www.bryanskobl.ru/economy-forecast-until-2019 (дата обращения 20.07.2021).

3. Новикова, А. В. Развитие электронной коммерции как фактор глобализации отечественной экономики / А. В. Новикова, О.И. Митина // Экономика в условиях социально-техногенного развития мира [Текст] + [Электронный ресурс]: материалы II Международной междисциплинарной научной конференции по фундаментальным и прикладным проблемам современного социально-экономического и экономико-экологического развития (5 октября 2017 г., г. Брянск, БГТУ совместно с РАН): в 2 т. / под ред. Е.А. Дергачевой. - Брянск: БГТУ, 2017. - Т.2. - 530 с. - С. 80-86.

4. Исайченкова, В. В. Использование современных информационных технологий при оценке бизнес-процессов промышленных предприятий / В. В. Исайченкова, С. П. Новиков // Конкурентоспособность в глобальном мире: экономика, наука, технологии. - 2017. - № 12 (59). - С. 310-312.

5. Новикова, А. В. Состояние и перспективы развития электронной торговли в Российской Федерации / А. В. Новикова, А. В. Крамарь // Вызовы цифровой экономики: условия, ключевые институты, инфраструктура: сборник статей I Всероссийской научно-практической конференции (г. Брянск, 21-22 марта 2018 г.) [Электронный ресурс]. Брянск: Брян. гос. инженерно-технол. унт. $2018 .-288$ c. - C. $245-249$. 
6. Борисенко, М. А. Логистическая инфраструктура как ключевое конкурентное преимущество современного предприятия / М. А. Борисенко, В. В. Исайченкова // Цифровая логистика - интегрированный подход: Материалы X Всероссийской научно-практической конференции. - Брянск, 2020. - C. 90-93.

7. Новикова, А. В. Формирование конкурентной стратегии промышленного предприятия на основе использования преимущественно процессно-ориентированного подхода: Дисс. на соискание степени к.э.н. Орёл, 2006. - 170 с.

8. Шпиленок А. Ю. Цифровизация как новый вектор экономического роста в России / А. Ю. Шпиленок, С. П. Новиков // Вызовы цифровой экономики: итоги и новые тренды. Сборник статей II Всероссийской научнопрактической конференции. - 2019. - С. 665-670.

9. Мхитарян, С. В. Маркетинговое управление поставками : учебное пособие / С. В. Мхитарян. - Москва : Евразийский открытый институт, 2016. - 136 с. - ISBN 978-5-374-00468-7. - Текст : электронный // Электронно-библиотечная система IPR BOOKS : [сайт]. — URL: http://www. iprbookshop.ru/10766.html. — Режим доступа: для авторизир. пользователей.

10. Палагин, Ю. И. Логистика - планирование и управление материальными потоками : учебное пособие / Ю. И. Палагин. - 2-е изд. Санкт-Петербург : Политехника, 2020. — 288 с. — ISBN 978-5-7325-1084-3. — Текст : электронный // Электронно-библиотечная система IPR BOOKS : [сайт]. — URL: http://www.iprbookshop.ru/94836.html. — Режим доступа: для авторизир. пользователей.

(C) А.В. Новикова, 2021 